\title{
Mullerian Duct Anomalies: Diagnostic Impact of Magnetic Resonance Imaging
}

\author{
Authors \\ Ola Mohamed Darwish ${ }^{1}$, Alaa Eldin Mohamed Mustafa ${ }^{2}$ \\ ${ }^{1}$ Department of Radio-Diagnosis, Hattah Hospital, Dubai Health Authority, Dubai, UAE \\ ${ }^{2}$ Department of Radio-diagnosis, Faculty of Medicine, Alexandria University, Egypt
}

\begin{abstract}
Aim: The aim of the present work is to evaluate the value of magnetic resonance imaging (MRI) in assessment of Mullerian duct anomalies.

Patients and Methods: The study was performed on 20 female patients having clinical gynecological complains and US imaging findings suggestive of having mullerian duct anomalies. All of them undergone clinical assessment, pelvic ultrasound, hysterosalpingography and non-contrast MRI of the pelvis.

Results: The study included 4 uterine agenesis, 2 uterine hypoplasia, 2 bicornuate uteri, 2 unicornuate uteri, 4 septate uteri, 4 uterine didelphys (associated with hemivaginal septum), 1 arcuate uterus and 1 with isolated complete vaginal septum. The circumstances leading to the diagnosis were 1ry amenorrhea (25\%), 1ry infertility (15\%), dysmenorrhea (15\%), recurrent abortion (15\%), menstrual irregularity (10\%), cyclic pelvic pain (5\%) while $15 \%$ were incidentally noted. US was done for all cases. It correctly diagnosed absent uterus in 4 cases with uterine agenesis and bicornuate uterus (in 2 cases). it incorrectly diagnosed the cases of uterine didelphys (4 cases) as bicornuate uterus. It missed the diagnosis of uterine hypoplasia, unicornuate uterus, septate uterus, arcuate uterus and vaginal septum. HSG was done for 10 cases. It correctly diagnosed a patient with unicornuate, 2 patients with bicornuate uterus \& patient with arcuate uterus. It misdiagnosed the cases of uterine didelphys as unicornuate uterus and the cases of septate uterus as bicornuate uterus. MRI was done for all cases. It succeeded to identify all patients with MDAs with proper specification of its types showing 100\% diagnostic accuracy.

Conclusion: Hystrosalpingography proved to be not suitable for complete assessment of MDAs. It couldn't be done to females with vaginal agenesis, vaginal septum and in patients with genital infection. Ultrasonography has limited capability in detection of double uterus, and cervical and vaginal anomalies. Not only MRI is sensitive in diagnosis of MDAs but also very accurate in MDAs specification. MRI is capable of detecting associated renal system anomalies.
\end{abstract}

Keywords: Mullerian duct anomalies (MDAs), MRI, HSG and ultrasound.

\section{Introduction}

The reported prevalence of MDA varies widely in the literature, ranging from $1 \%-5 \%$ in the general population to $13 \%-25 \%$ among women with recurrent pregnancy loss.

Fusion of the müllerian ducts normally occurs between the 6th and 11th weeks of gestation to form the uterus, fallopian tubes, cervix, and proximal two-thirds of the vagina. Any disruption of müllerian duct development during embryogenesis can result in a broad and complex spectrum of congenital abnormalities termed müllerian duct anomalies (MDAs). MDAs are not associated with anomalies of the external genitalia 
or ovarian development. Diagnosis of MDAs is clinically important because of the high associated risk of infertility, endometriosis, and miscarriage. MDAs are also commonly associated with renal anomalies, with a reported prevalence of 30\%$50 \%$, including renal agenesis (most commonly unilateral agenesis), ectopia, hypoplasia, fusion, malrotation, and duplication. ${ }^{(1-4)}$

Selection of the initial imaging modality is often dictated by the presenting clinical scenario (eg, primary amenorrhea, pelvic pain, or infertility). Hysterosalpingography (HSG) is routinely used in an initial evaluation of infertility; it allows assessment of the uterine cavity and fallopian tube patency but does not provide any information about the external uterine contour. In younger patients or acute cases, ultrasonography (US) is the preferred method because it is readily available, inexpensive, and rapid and does not use ionizing radiation. Field-of-view restrictions with US, patient body habitus, and artifact from bowel gas may result in a request for further imaging with MR imaging. With the advent of threedimensional (3D) techniques, US may have the future potential to match the capabilities of MR imaging. Currently, however, MR imaging remains the preferred MDA imaging method, as it exquisitely details both the uterine cavity and external contours and has shown excellent agreement with clinical MDA subtype diagnosis. ${ }^{(5-11)}$

In this study, we followed the classification of mullerian duct anomalies of American society for reproductive medicine. ${ }^{(13)}$ (table1)

Table (1): Classification of mullerian duct anomalies ${ }^{(13)}$

\begin{tabular}{|l|}
\hline Agenesis (vaginal, cervical, fundal, tubal, combined) \\
\hline poplasiaHy \\
\hline Unicornuate: \\
\hline 1 - Rudimentary horn \\
\hline -With endometrium \\
\hline a - communicating \\
\hline b- non communicating \\
\hline -Without endometrium \\
2- No rudimentary horn \\
\hline Uterus didelphys \\
\hline Bicornuate \\
\hline 1-Bicollis \\
\hline 2-Unicollis \\
\hline Septate \\
\hline 1- Complete \\
\hline 2- Incomplete \\
\hline Arcuate \\
\hline Diethylstilbestrol-drug related Anomalies (DES) \\
\hline Vaginal septum \\
\hline 1- Complete transverse septum \\
\hline 2 - Incomplete septum \\
\hline
\end{tabular}

\section{Aim of the Work}

The aim of the present work is to evaluate the value of magnetic resonance imaging (MRI) in assessment of Mullerian duct anomalies.

\section{Patients and Methods}

The present work included 20 female patients with gynecological complains, provisionally suggested to have mullerian duct anomalies on clinical and US basis. 
All the studied patients were subjected to complete history taking, physical examination, ultrasound examination (transabdominal and/or transvaginal), hysterosalpingography in 10 married female cases and non-contrast MRI study of the pelvis in all cases. The medical ethics were considered, the patients were aware of the benefits and complications and a written informed consent was taken before examination.

Ultrasound examination was done on a Siemens X300 ultrasound machine (Erlangen, Germany); including a convex probe for the abdominopelvic approach $(5.2 \mathrm{MHz})$ and endovaginal probe for the transvaginal approach $(9.4 \mathrm{MHz})$ in the 10 married female patients.

\section{Hysterosalpingography}

examination:

A

conventional radiograph of the pelvis was done before the contrast medium was administrated into the uterine cavity; so that possible masses or calcifications will not complicate interpretation of the images. The examination was performed under fluoroscopic control, radiographs were taken at filling phase of the uterine cavity (usually $2-3 \mathrm{~cm}^{3}$ of contrast medium were sufficient) and again after filling of the fallopian tubes. Finally, the presence of contrast medium in the peritoneal cavity was checked. Additional spot radiographs were obtained to document abnormality detected.

MRI sequences were acquired on closed magnet MRI machine Philips Gyroscan Intra (Netherlands, Eindhoven,) 1.5-tesla at the MRI unit. Analysis of the images was performed on specialized Philips medical system work station (Extended MR workspace R 2.6.3.1 soft ware).

All patients were examined in the supine position. All sequences were acquired with saturation bands placed anteriorly and posteriorly to eliminate the high signal from subcutaneous fat. Sedation by oral chloral hydrate $(50-70 \mathrm{mg} / \mathrm{kg}$ body weight, 20-30 minutes before examination) was done to the single infant included in the study.

The MRI examination included sagittal T2weighted fast spin-echo sequence from one femoral head to the other, oblique axial T2weighted fast spin-echo sequence from the renal hilum to the symphysis pubis, oblique axial T1weighted spin-echo sequence (perpendicular to the long axis of the uterus), and oblique Coronal T2 weighted sequence (parallel to the long axis of the uterus). The acquisition protocols, including sequences and parameters are shown in table (2).

Table (2): Parameters of MR sequences

\begin{tabular}{|l|c|c|c|c|c|}
\hline & Sagittal T2 & Axial T2 & Coronal T2 & Axial T1 & $\begin{array}{c}\text { Fast spin echo T2W } \\
\text { images }\end{array}$ \\
\hline TR/TE & $4510 / 96$ & $5210 / 71$ & $4000 / 94$ & $2470 / 10$ & $1800 / 100$ \\
\hline Echo train length & 13 & 14 & 13 & 13 & - \\
\hline Slice thickness & 3.5 & 5.5 & 6 & 5.5 & 3.0 \\
\hline Gap & 5.4 & 6 & 7.2 & 6 & 3.0 \\
\hline Field of view & $280 \times 280$ & $360 \times 360$ & $350 \times 350$ & $360 \times 360$ & $360 \times 360$ \\
\hline Matrix size & $205 \times 256$ & $256 \times 320$ & $460 \times 350$ & $288 \times 320$ & $150 \times 512$ \\
\hline Flip angle & 150 & 150 & 150 & 150 & 150 \\
\hline
\end{tabular}

\section{Results}

The present work included 20 female patients with gynecological complains, provisionally suggested to have mullerian duct anomalies on clinical and US basis. Their ages ranged from 7 months to 47 years with a mean age of 24.6 years. The most commonly included age group was the 3rd decade followed by the 4 th decade. 
The 20 patients included in the study sought medical advice due to 1ry amenorrhea in 5 patients, 1ry infertility in 3 patients, dysmenorrhea in 3 patients, recurrent abortion in 3 patients, menstrual irregularities in 2 patients and cyclic pelvic pain in 1 patient. In the remaining 3 patients, the pathology was incidentally discovered on US basis.

\section{Diagnostic yields of different imaging techniques in 20 patients with MDAs (table 3)}

Table (3): Comparison between MRI, US \& HSG in diagnosis of 20 patients with MDAs

\begin{tabular}{|c|c|c|c|c|}
\hline Types of MDA & $\begin{array}{l}\text { Number of } \\
\text { patients }\end{array}$ & MR Diagnosis & US diagnosis & $\begin{array}{c}\text { Hystrosalpin-gographic } \\
\text { Diagnosis (done for } 10 \text { cases) }\end{array}$ \\
\hline Agenesis & 4 & $4 / 4$ & $4 / 4$ & Not done \\
\hline Hypoplasia & 2 & $2 / 2$ & $0 / 2$ & Not done \\
\hline Unicornuate & 2 & $2 / 2$ & $0 / 2$ & $1 / 1$ \\
\hline $\begin{array}{l}\text {-Rudimentary horn with non } \\
\text { communicating endometrium. } \\
\text {-No rudimentary horn }\end{array}$ & $\begin{array}{l}2 \\
-\end{array}$ & $\begin{array}{l}2 / 2 \\
-\end{array}$ & $0 / 2$ & $\begin{array}{l}0 / 1 \\
1 / 1\end{array}$ \\
\hline Uterus didelphys & 4 & $4 / 4$ & $0 / 4$ & $2 / 2$ \\
\hline Bicornuate & 2 & $2 / 2$ & $2 / 2$ & $2 / 2$ \\
\hline Bicollis & 1 & $1 / 1$ & $\mathbf{0} / \mathbf{1}$ & $1 / 1$ \\
\hline Unicollis & 1 & $1 / 1$ & $\mathbf{0} / \mathbf{1}$ & $1 / 1$ \\
\hline Arcuate & 1 & $1 / 1$ & 0/1 & $1 / 1$ \\
\hline Septate & 4 & $4 / 4$ & $0 / 4$ & $0 / 4$ \\
\hline 1- Complete & 1 & $1 / 1$ & $\mathbf{0} / \mathbf{1}$ & $\mathbf{0} / \mathbf{1}$ \\
\hline 2 - Incomplete & 3 & $3 / 3$ & $0 / 3$ & $0 / 3$ \\
\hline Vaginal septum: & 5 & $5 / 5$ & $0 / 5$ & Not done \\
\hline $\begin{array}{l}\text { 1- } \begin{array}{l}\text { Complete transverse } \\
\text { septum }\end{array} \\
2 \text { - Incomplete septum }\end{array}$ & $\begin{array}{l}1 \\
4\end{array}$ & $\begin{array}{l}1 \\
4\end{array}$ & $\begin{array}{l}0 / 1 \\
0 / 4\end{array}$ & \\
\hline
\end{tabular}

\section{US diagnosis of MDAs}

US correctly diagnosed the absence of the uterus in the four patients with uterine agenesis. It also correctly diagnosed bicornuate uterus (figure 1) in 2 patients with detection of large indentation in the uterine fundus, divergent uterine horns, and echogenic endometrial complexes. However in both above mentioned cases, it failed to differentiate between bicornuate bicollis and bicornuate unicollis anomaly as it couldn't identify the cervical component of the anomaly.

US failed to diagnose uterine hypoplasia in 2 patients (which is defined if the distance between the cornu is less than $2 \mathrm{~cm}$ or if the distance between the internal os to the fundus is less than 3 to $5 \mathrm{~cm}$ ) as evaluation of a uterine remnant was difficult with US owing to the limited acoustic window. Furthermore, unicornuate uterus (2 cases), arcuate uterus (1 case) and septate uterus ( 4 cases) were misinterpreted as normal uterus by US. It also misdiagnosed uterine didelphys (4 cases) (figure 2,3) as bicornuate uterus; as it failed in description of associated defect including complete duplication of the vagina and cervix. It correctly diagnosed unilateral renal agenesis that was present in three out of four patients with uterine didelphis. It also failed to identify the longitudinal hemivaginal septum that was present 
in these four patients. US also couldn't identify the septum in the single case presenting with isolated vaginal septum, however it could only detect hematometra and hematocolpos in this case and suggested no explanation for fluid retention.

\section{HSG diagnosis of MDAs}

HSG was done in only 10 out of 20 patients suffering from MDAs as the other 10 patients were virgin. HSG was done in the following distribution; in one out of two patients suffering from unicornuate uterus, two out of four patients suffering from uterine didelphys, all patients suffering from bicornuate uterus (two patients), all patients suffering from septate uterus (four patients) and in the single patient suffering from arcuate uterus.

HSG succeeded in identification of the unicornuate uterine anomaly in one single by identification of an off midline fusiform uterine cavity with contrast opacification of a solitary fallopian tube, but it failed to detect the rudimentary horn (as it was non communicating).

HSG correctly detected bicornuate uterus in two patients (figure 1) with opacification of two symmetric fusiform uterine cavities (horns) and fallopian tubes with proper identification of its two varieties whether bicollis (one patients) or unicollis (the other patient).

HSG successfully identified the arcuate uterus anomaly in the single patient presented with this anomaly, with identification of single uterine cavity and a broad saddle-shaped indentation at the uterine fundus.

HSG misinterpreted the anomaly in the two patients with uterine didelphys (figure 2,3) as unicornuate unicollis uterus yet it failed in demonstration of longitudinal hemivaginal septum with opacification of single uterine cavity and a solitary fallopian tube in both cases .

HSG misinterpreted the anomaly in the four patients with septate uterus as bicornuate bicollis uterus. It identified two uterine cavities, however it does not provide any information about the external uterine contour or the myometrium.
MRI diagnosis of MDAs

MRI succeeded in correct diagnosis of all patients presented with uterine hypoplasia (2 cases) depending upon demonstration of abnormally exaggerated low-signal-intensity myometrium on T2-weighted images, and an endometrial layer. Uterine agenesis was also correctly diagnosed in 4 patients showing complete absence of the uterus. Unicornuate uterus with non communicating rudimentary horn showing endometrium was also detected by MRI in 2 patients, the anomaly expressed banana-shaped (curved and elongated) uterus with the functional non-communicating horn appearing as a cavity deformed by the enlarged rudimentary horn, which had a highsignal-intensity center on both T1and T2weighted images; a finding that was compatible with hematometra. MRI also correctly diagnosed uterine didelphys (4 cases) (figure 2,3) showing widely divergent uterine horns, with a deep midline fundal cleft affecting the external contour of the uterine fundus as well as two separate cervices, and a longitudinal septum in the upper vagina associated with hematometrocolpos with varying degrees of distention within the obstructed hemivagina.

Bicornuate uterus was also successfully diagnosed by MRI in 2 cases (figure 1) with identification of two separate uterine horns and a deeply notched uterine fundus; the external cleft was more than 1 $\mathrm{cm}$ in length; one of the cases was bicornuate bicollis with the septum extended to the external os still with some degree of communication between the two horns; the other case expressed bicornuate unicollis anomaly with the septum extended to the internal os.

Arcuate uterus was diagnosed also by MRI (1 case) with normal external contour and single uterine cavity with a broad saddle-shaped indentation at the uterine fundus. MRI also diagnosed septate uterus (in 4 patients), it revealed a normal-sized uterus, with the endometrial cavity appearing smaller than a normal cavity; three cases were incomplete septate with partial division of the endometrial canal by a solid mass 
demonstrating signal intensity similar to that of the adjacent myometrium that extends to the internal cervical os and the remaining case had complete septate configurations with the septum seen extending to the external cervical os. The inferior segment of the septum of complete septate uterus was composed of fibrous tissue appearing as a low-signal-intensity linear band extending to the external cervical os on T2weighted images.
MRI also correctly identified vaginal septum in 5 cases. The septum appeared as thin, low-signalintensity structure that was best visualized in the coronal and axial planes; one case presented with complete vaginal septum (isolated complete transverse vaginal septum) and the remaining four cases were incomplete (longitudinal hemivaginal septum and associated with uterine didelphys). (figures $2 \& 3$ )

\section{Illustrative cases}

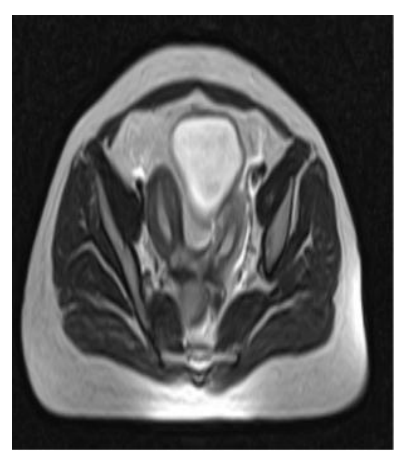

C

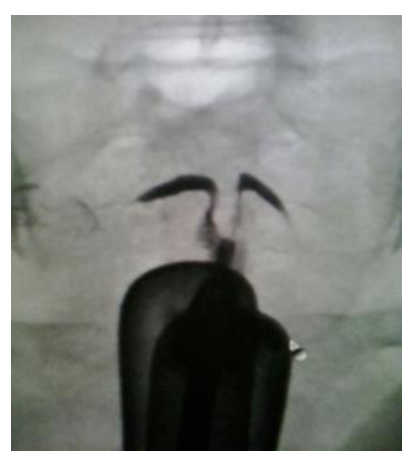

B

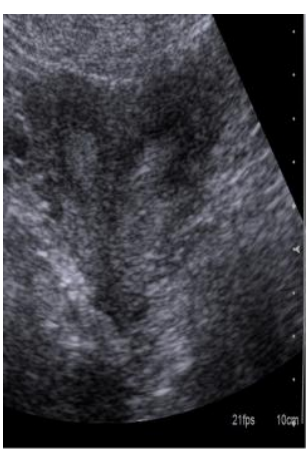

A

Figure (1): A 24 years old patient with bicornuate unicollis. A) Axial oblique T2 MR image showing the two uterine horns and single cervix. B) HSG: Injection of contrast into the cervix revealed bicornuate unicollis uterus with opacification of the cervix and the two uterine horns C) Axial TVUS image showing bicornuate uterus with a large indentation in the uterine fundus and divergent uterine horns.

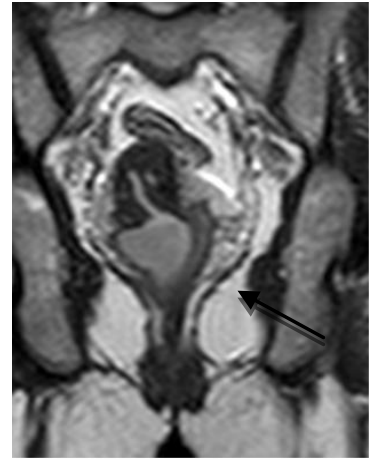

A

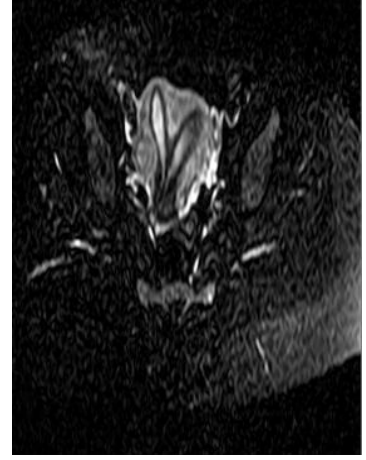

B

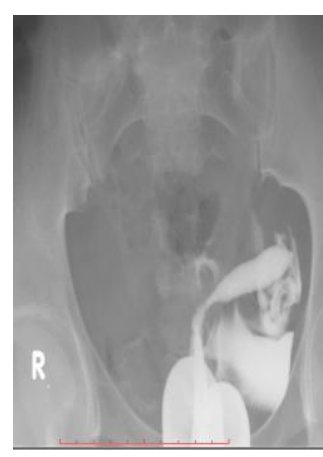

C



D

Figure (2): 33 ys patient with didelphys uterus A) Coronal T2 MR image showing right hematocolpos due to right hemivaginal septum (black arrow) B) Axial T2 fat suppressed MR image in the same patient revealed double uterine cavities. B) HSG: Injection of contrast through the left hemivagina into the left cervix revealed opacification of the left uterine cavity and left fallopian tube with wrong diagnosis of the case as unicornuate uterus C) Axial transvaginal US image showing large indentation in the uterine fundus, divergent uterine horns, and echogenic endometrial complexes wrongly diagnosed as bicornuate uterus. 


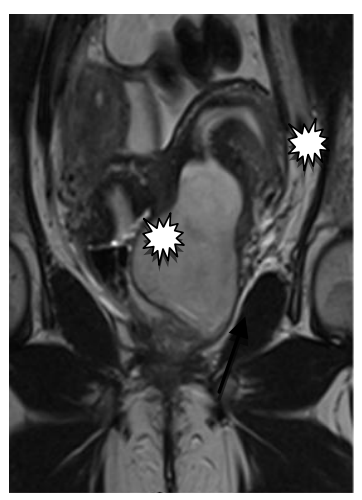

A

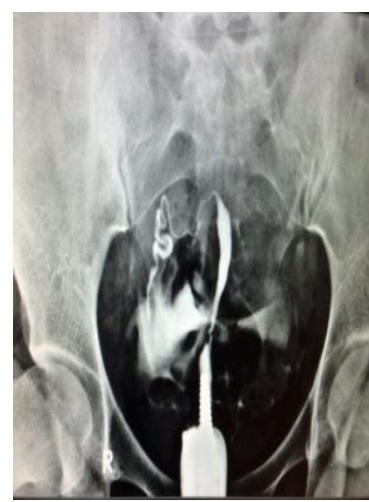

B

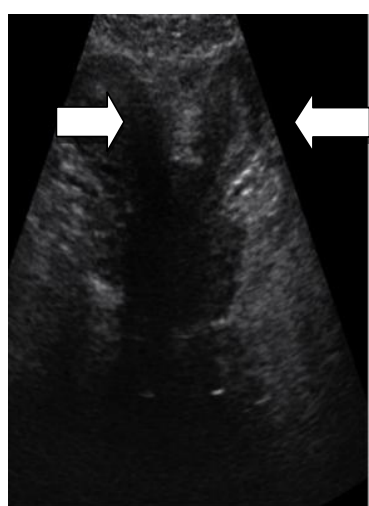

C

Figure (3): 20 ys patient with didelphys uterus A) Coronal T2 MR image showing double uterine cavities , double cervices (white asterix) with no communication in between, with with left hemivaginal septum (black arrow) and left hematocolpos. B) HSG: Injection of contrast through the right hemivagina into the right cervix revealed opacification of the right uterine cavity and right fallopian tube with wrong diagnosis of the case as unicornuate uterus C) Axial US image shows large indentation in the uterine fundus, divergent uterine horns (white arrows), and echogenic endometrial complexes wrongly diagnosed as bicornuate uterus.

\section{Discussion}

This study was performed in attempt to evaluate the role of MRI in characterization of MDAs trying to compare its yield with that of US and HSG.

Primary amenorrhea was the presenting symptom in patients with agenesis and hypolplasia. ${ }^{(1)}$ This agree with the present study which included 6 patients (agenesis $=4$, hypoplasia $=2$ ), 5 of them presented with primary amenorrhea and the remaining 1 patient (infant 7 months) was incidentally detected by US assessment.

In the current study, 6 patients presented with complete uterine agenesis and uterine hypoplasia, all of them were correctly diagnosed by MRI. This agree with Behr ${ }^{(1)}$ who reported that MRI can fulfill the diagnosis of theses anomalies because of its inherent ability to detect the uterus, cervix and vagina depending upon their specific signal intensities and due to multiplanar capability of MRI. Uterine hypoplasia and agenesis were best appreciated in the sagittal plane. However, vaginal agenesis is best characterized on axial images. In uterine hypoplasia, an exaggerated low-signalintensity of the myometrium is typically seen on T2-weighted images with an endometrial layer. They also reported that the functional uterine remnant presents as poorly differentiated zonal anatomy and reduced endometrial and myometrial width. Features which were identified in all patients having the anomaly included in the current study group. Junqueira ${ }^{(4)}$ also relied also on these signs to diagnose uterine hypoplasia.

In the present study; both MRI and US had the same successful diagnostic ability in all four patients having uterine agenesis. This disagree with $\mathrm{Behr}^{(1)}$ who reported that MRI is superior to US in diagnosis of agenesis.

The present study revealed that MRI is superior to US in diagnosis of 2 patients with uterine hypoplasia. These patients were not candidates for hystrosalpingography. This agrees with the study of $\mathrm{Behr}^{(1)}$. He relied upon the demonstration of any functioning endometrial tissue by MRI to diagnose hypoplastic uterus which was difficult to be detected by US.

Unicornuate uterus accounts for $6-25 \%$ of all MDAs. ${ }^{(13,14)}$ In the current study the anomaly accounted for $10 \%$ of all MDAs.

MRI was superior to both US and HSG in detection of all patients with unicornuate uterus included in the present study, as MRI could detect the main uterine cornu and also the associated non communicating rudimentary horn whereas rudimentary horn could be detected by US. Also the rudimentary horn could be detected by HSG as 
it was non communicating horn. These agree with $\mathrm{Behr}^{(1)}$ who reported that MRI is more accurate than US and HSG for diagnosis of the unicornuate uterus.

Didelphys uterus accounts for $5-11 \%$ of all MDAs ${ }^{(13,14)}$. This disagrees with the current study where uterine didelphys accounted for $20 \%$ of the study group. This might be attributed to the smaller number of patients included in the current study (20 cases compared to 144 cases in Buttram study and 29 cases in Carrington study). In the current study, MRI was superior to US and HSG for diagnosis of uterus didelphys.

This agrees with $\mathrm{Behr}^{(1)}$ who attributed the findings to MRI inherent ability to detect the uterus, cervix and vagina due to their specific signal intensities as well as the multiplanar capability of MRI aiding in detection of partial or complete duplication involving the uterus, cervix and vagina.

Bicornuate uterus accounts for $10-39 \%$ of all $\operatorname{MDAs}^{(13,14)}$. The current study also revealed that unicornuate uterus accounts for $10 \%$.

It has been reported that septate uterus accounts for $34-55 \%$ of all MDAs ${ }^{(13,14)}$. An incidence which does no match with the current study which revealed that septate uterus accounted for $20 \%$. This is may be attributed to smaller number of the cases included in the current study compared to those included in Buttram and Carrington study $(13,14)$

The current study showed that MRI is was superior to US and HSG in differentiation between bicornuate and septate uteri. The three important signs to distinguish between the two anomalies (fundal contour, type of the septum and if the apex of the external fundal contour is above or below the line drawn between the uterine ostia) were well demonstrated by MRI due to its multiplanar capability and good delineation of the anatomical structures with specific signal intensities. These signs were difficult to be applied by US or HSG.

Janqueira $^{(4)}$ reported that applying these signs using MRI, precise diagnosis is always obtained.
Reuter $^{(12)}$ reported that US and HSG should be used together to get a diagnosis of bicornuate and septate uterus more accurate than could be obtained if each technique was performed alone.

The first sign was the shape of the upper surface of the uterine fundus. It is flat or convex in septate uterus while in bicornuate uterus, it is concave. ${ }^{(1)}$ In the current study, the four patients with septate uterus showed flat surface and the two patients with bicornuate uterus showed concave surface. On the other hand Monica Epelman ${ }^{(3)}$ found this sign to be misleading because the external fundal contour in septate uterus may be convex, flattened or mildly concave (with the fundal cleft measuring $<10 \mathrm{~mm}$ ).

The second sign was the thickness and signal intensity of the septum between two endometrial cavities. If the septum is thick and of the same signal intensity like that of the myometrium, bicornuate uterus was diagnosed ${ }^{(3)}$. This sign was found in the two patients presented with bicornuate uterus in the current study. While the inferior segment of a complete septate uterus showed low signal intensity on $\mathrm{T} 1$ and $\mathrm{T} 2$ weighted images, which meant it is a fibrous septum that was found in two out of four patients with septate uterus. This agrees with Monica Epelman $^{(3)}$ who relied on this sign as one of the signs used to differentiate between these anomalies. On the other hand, Junqueira et $\mathrm{al}^{(4)}$ and $\mathrm{Behr}$ et $\mathrm{al}^{(1)}$ reported a different concept considering the composition of the uterine septum between the uterine horns not to be a differentiating feature since fibrous tissue and/or myometrium may be found in both entities and this sign cannot differentiate between partial septate and bicornuate uterus.

The third sign is a line drawn between the uterine ostia of fallopian tubes; if the apex of the external fundal contour is more than $5 \mathrm{~mm}$ above this line, septate uterus is diagnosed, while in bicornuate or didelphys uterus, the apex of the external fundal contour lies below or less than $5 \mathrm{~mm}$ above the interostial line. ${ }^{(1)}$ These group of anomalies included in the current study showed the same 
sign in all cases ( including 10 cases: 4 sepatate, 4 didelphys and 2 bicornuate anomalies).

It has been reported that the above three signs should be used to differentiate accurately between the bicornuate and septate anomalies because of the difference in methods of management and their prognosis as bicornuate uterus is not amenable to surgical intervention, while septate uterus can be effectively treated with hysteroscopic resection of the septum leading to improved obstetric outcome ${ }^{(4)}$.

Arcuate uterus accounted for $5 \%$ of the anomalies included in the current study, this agrees with the other reported incidence of $7 \%$ of all MDAs $(13,14)$

In the current study, MRI and HSG were superior to US for diagnosis of arcuate uterus as the shape of the endometrial cavity could be identified clearly by these techniques.

This disagrees with $\mathrm{Behr}^{(1)}$ who reported that US, HSG and MRI could detect arcuate uterus. Behr depended in their study upon the shape of the endometrial cavity to diagnose arcuate uterus.

In the current study, MRI was superior to US for diagnosis of vaginal septum, including one case with isolated complete vaginal and 4 cases of longitudinal hemivaginal septum and associated with uterine didelphys. MRI succeeded in demonstration of the obstructing cause (septum) depending upon its different signal intensity and also due to its capability to demonstrate the vagina. Janqueria ${ }^{(4)}$ also reported that MRI is superior to US as it enables accurate detection of the presence of a vaginal septum by its different signal intensity. In the current study, patients having this anomaly were not candidates for hystrosalpingography.

\section{Conclusions}

Hystrosalpingography is the traditional method of identifying anomalies of the reproductive system. It is helpful in demonstrating tubal patency and intra uterine adhesions but it necessitates exposure to contrast material and ionizing radiation. However, hystrosalpingography is not a suitable technique complete assessment of MDAs. It couldn't be done in virgin females, females with vaginal agenesis, vaginal septum and in patients with genital infection.

Ultrasonography is being the initial diagnostic modality in examination of patients suspected to have Mullerian duct anomalies. It offers the lowest cost with no risk of ionizing radiation. Although it can detect some of the Mullerian duct anomalies, it has limited capability in detection of others including double uterus, cervical and vaginal anomalies.

Not only MRI is sensitive in diagnosis of MDAs but also very accurate in their specification which serves the proper management. MRI can be used in the same setting to study the renal system in which anomalies are more common in association with MDAs.

\section{References}

1. Behr SC, Courtier JL, Qayyum A. Imaging of Müllerian Duct Anomalies. Radiographics 2012; 32(6):233-50.

2. Gouhar GK, Siam S. Uterine septum structure and reproductive performance: Role of 3D TVUS and MRI. Egyptian J Radiol Nuclear Med 2013; 44, 357-365

3. Epelman M, Dinan D, Gee MS et-al. Mullerian duct and related anomalies in children and adolescents. MagnReson Imaging Clin N Am 21 (2013) 773-789.

4. Junqueira BL, Allen LM, Spitzer RF et-al. Müllerian Duct Anomalies and Mimics in Children and Adolescents: Correlative Intraoperative Assessment with Clinical Imaging1. Radiographics 2009; 29(4):1085103.

5. Olpin JD, Heilbrun M. Imaging of Müllerian duct anomalies. Topics in Magnetic Resonance Imaging 2010; 21(4):225.

6. Deutch TD, Abuhamad AZ. The Role of 3Dimensional Ultrasonography and Magnetic Resonance Imaging in the Diagnosis of Müllerian Duct Anomalies A Review of the 
Literature. Journal of Ultrasound in Medicine 2008; 27(3):413-23.

7. Shulman Leep. Mullerian anomalies. Clinical obstetrics and gynecology 2008; 51(2):214-22.

8. Mazouni C, Girard G, Deter R et-al. Diagnosis of Mullerian anomalies in adults: evaluation of practice. Fertility and sterility 2008; 89(1):219-22.

9. Edmonds DK. Rokitansky syndrome and other Mullerian anomalies. In: Balen AH, Creighton SM, Davies MC, MacDougall J, Stanhope R, eds. Paediatric and adolescent gynaecology. Cambridge, England: Cambridge University Press, 2004; 267274.

10. The American Fertility Society classifications of adnexal adhesions, distal tubal occlusion, tubal occlusion secondary to tubal ligation, tubal pregnancies, müllerian anomalies and intrauterine adhesions. FertilSteril 1988;49(6):944-955.

11. Troiano RN, McCarthy SM. Müllerian duct anomalies: imaging and clinical issues. Radiology 2004; 233(1):19-34.

12. Reuter K, Daly Dand Cohen S: Septate versus bicornuate uteri: errors in imaging diagnosis. Radiology 1998:172:749-752.

13. Buttram VC, Gibbons WE. Müllerian anomalies: a proposed classification. (An analysis of 144 cases). Fertil. Steril. 1979;32 (1): 40-6.

14. Carrington BM, Hricak H, Nuruddin RN etal. Mullerian duct anomalies: MR imaging evaluation. Radiology. 1990;176 (3). 